\title{
Fundamentos para un currículo nacional en Cirugía General basado en competencia: Consenso Delphi de la División de Educación de la Asociación Colombiana de Cirugía
}

\author{
Foundations for a national competence-based curriculum in General
} Surgery: Delphi Consensus of the Division of Education of the Colombian Association of Surgery

\author{
Luis Carlos Domínguez $\mathbb{D}^{\mathbb{D}}$, Álvaro Enrique Sanabria² ${ }^{\mathbb{D}}$, Lilian Torregrosa-Almonacid ${ }^{3} \mathbb{D}$, \\ William Sánchez $\mathbb{D}^{\mathbb{D}}$, Neil Valentín Vega ${ }^{5} \mathbb{D}$, Felipe Vargas ${ }^{6} \mathbb{D}$, Álvaro Niño $^{7}$, Liliana Cuevas ${ }^{8} \mathbb{D}$, \\ Sergio Iván $\operatorname{Hoyos}^{9} \mathbb{D}$, Luis Gerardo Cadavid ${ }^{10} \mathbb{D}$, Germán Jiménez ${ }^{11}$, Julián Sarmiento ${ }^{12} \mathbb{D}$, \\ Mauricio Osorio ${ }^{13}$, Maikel Pacheco ${ }^{14} \mathbb{D}$, Rubén Caycedo ${ }^{15}$, Rolando Medina ${ }^{16} \mathbb{D}$, Jimmy León ${ }^{17} \mathbb{D}$, \\ Mauricio Zuluaga ${ }^{18} \mathbb{D}$, Hernando Coba ${ }^{19}$, Erwin Rodríguez ${ }^{20}$, Carlos Luna-Jaspe ${ }^{21} \mathbb{D}$, \\ Jaime Merlano ${ }^{22}$, Juan Jacobo Molina ${ }^{23}$ (D), Carlos Bustillo ${ }^{24}$
}

1 Médico, especialista en Cirugía general, doctor en Educación para Profesiones de la Salud; presidente, División de Educación, Asociación Colombiana de Cirugía; profesor asociado, Departamento de Cirugía, Universidad de la Sabana, Chía, Colombia.

2 Médico, especialista en Cirugía general, magister en Epidemiología Clínica y Economía de la Salud, doctor en Oncología de Cabeza y Cuello, CEXCA (Centro de Excelencia en Enfermedades de Cabeza y Cuello); profesor titular, Departamento de Cirugía, Facultad de Medicina, Universidad de Antioquia, Medellín, Colombia

3 Médico, FACS, especialista en Cirugía general, Cirugía de Seno y Tejidos Blandos y magíster en Bioética; profesor titular, Directora Departamento de Cirugía y Especialidades, Facultad de Medicina, Pontificia Universidad Javeriana, Hospital Universitario San Ignacio. Presidente, Asociación Colombiana de Cirugía, Bogotá, D.C., Colombia.

4 Médico, especialista en Cirugía general; Director ejecutivo, Asociación Colombiana de Cirugía; jefe, Departamento de Cirugía, Hospital Militar Central, Bogotá, D.C, Colombia.

5 Médico, especialista en Cirugía general; jefe, Departamento de Cirugía, Universidad de la Sabana, Chía, Colombia.

6 Médico, especialista en Cirugía general; jefe, Departamento de Cirugía, Universidad del Rosario, Bogotá, D.C, Colombia.

7 Médico, especialista en Cirugía general; jefe, Departamento de Cirugía, Universidad Industrial de Santander, Bucaramanga, Colombia.

8 Médica, especialista en Cirugía general; profesora, Departamento de Cirugía, Pontificia Universidad Javeriana, Bogotá, D.C, Colombia.

9 Médico, especialista en Cirugía general; jefe, Departamento de Cirugía, Universidad Pontificia Bolivariana, Medellín, Colombia.

10 Médico, especialista en Cirugía general; jefe, Departamento de Cirugía, Universidad CES, Medellín, Colombia.

11 Médico, especialista en Cirugía general; jefe, Departamento de Cirugía, Fundación Universitaria Sanitas, Bogotá, D.C, Colombia.

12 Médico, especialista en Cirugía general; jefe, Departamento de Cirugía, Universidad del Cauca, Popayán, Colombia.

13 Médico, especialista en Cirugía general; jefe, Departamento de Cirugía, Universidad de Caldas, Manizales, Colombia.

14 Médico, especialista en Cirugía general; jefe, Departamento de Cirugía, Fundación Universitaria Ciencias de la Salud, Bogotá, D.C, Colombia.

15 Médico, especialista en Cirugía general; jefe, Departamento de Cirugía, Universidad Nacional de Colombia, Bogotá, D.C, Colombia.

16 Médico, especialista en Cirugía general; jefe, Departamento de Cirugía, Universidad Surcolombiana, Neiva, Colombia.

17 Médico, especialista en Cirugía general; jefe, Departamento de Cirugía, Universidad de Antioquia, Medellín, Colombia.

18 Médico, especialista en Cirugía General y Mínimamente Invasiva; jefe, Sección de Cirugía General, Universidad del Valle; jefe, Unidad de Cirugía Bariátrica y de Pared Abdominal, Hospital Universitario del Valle, Cali, Colombia.

19 Médico, especialista en Cirugía general; jefe, Departamento de Cirugía, Universidad de Cartagena, Cartagena, Colombia.

20 Médico, especialista en Cirugía general; jefe, Departamento de Cirugía, Universidad Militar Nueva Granada, Bogotá, D.C, Colombia.

21 Médico, especialista en Cirugía general; director, Especialización en Cirugía General, Universidad El Bosque; jefe, Departamento quirúrgico, Los Cobos Medical Center, Bogotá, D.C, Colombia.

22 Médico, especialista en Cirugía general; jefe, Departamento de Cirugía, Universidad Libre, Barranquilla, Colombia.

23 Médico, especialista en Cirugía general; jefe, Departamento de Cirugía, Universidad Metropolitana, Barranquilla, Colombia.

24 Médico, especialista en Cirugía general; jefe, Departamento de Cirugía, Universidad del Sinú, Cartagena, Colombia.

Fecha de recibido: 15/02/2021 - Fecha de aceptación: 26/03/2021 - Fecha de publicación en línea: 03/06/2021

Correspondencia: Luis Carlos Domínguez, Campus del Puente del Común, Km. 7, Autopista Norte de Bogotá, Chía, Colombia.

Teléfono: 8615555 / 861 6666. Dirección electrónica: carlosdot@unisabana.edu.co.

Citar como: Domínguez LC, Sanabria AE, Torregrosa-Almonacid L, Sánchez W, Vega NV, Vargas F, et al. Fundamentos para un currículo nacional en Cirugía General basado en competencia: Consenso Delphi de la División de Educación de la Asociación Colombiana de Cirugía. Rev Colomb Cir. 2021;36:582-98. https://doi.org/10.30944/20117582.898

Este es un artículo de acceso abierto bajo una Licencia Creative Commons - BY-NC-ND https://creativecommons.org/licenses/by-nc-nd/4.0/deed.es 


\title{
Resumen
}

Frente a los desafíos de la Educación Basada en Competencia, este consenso Delphi de la División de Educación de la Asociación Colombiana de Cirugía informa sobre las mínimas competencias profesionales esperadas del cirujano egresado de los veinte programas de Especialización en Cirugía General en Colombia. Un total de 105 profesores de los programas de especialización evaluaron tres áreas de competencia profesional: 1) atributos profesionales generales del residente durante su formación, 2) competencias prácticas (procedimientos quirúrgicos) que los residentes deben realizar al final de su entrenamiento y 3) Actividades Profesionales Confiables (APC) que los residentes deben ejecutar sin supervisión al final de su entrenamiento. Los resultados informan un alto nivel de consenso en el $100 \%$ los atributos profesionales generales y APC, y del $75 \%$ en diferentes procedimientos quirúrgicos. El consenso abre la puerta para el desarrollo de un currículo nacional de la especialidad y tiene implicaciones para la práctica educativa e investigación futura.

Palabras clave: cirugía general; programas de postgrado; currículo; educación basada en competencia; consenso; Delphi; Colombia.

\begin{abstract}
Faced with the challenges of Competence-Based Education, this Delphi consensus from the Education Division of the Colombian Association of Surgery reports on the minimum professional competencies expected of the surgeon who graduated from the twenty residency programs in General Surgery in Colombia. A total of 105 professors from the training programs evaluated three areas of professional competence: 1) general professional attributes of the resident during their training, 2) practical skills (surgical procedures) that residents must perform at the end of their training, and 3) Activities Trusted Professionals (APC) that residents must run unsupervised at the end of their training. The results report a high level of consensus in 100\% general professional attributes and APC, and $75 \%$ in different surgical procedures. The consensus opens the door for the development of a national specialty curriculum and has implications for educational practice and future research.
\end{abstract}

Keywords: general surgery; postgraduate programs; curriculum; competence-based education; consensus; Delphi; Colombia.

\section{Introducción}

La transformación acelerada de los sistemas educativo y sanitario ha traído cambios en la formación médica a nivel global. Los principales tienen que ver con la introducción progresiva del enfoque de Educación basada en Competencia (EBC) y dominio del aprendizaje, y de sistemas de evaluación del desempeño profesional integral.

La EBC, en particular, es un enfoque de educación basada en resultados, dirigido a la obtención de un nivel definido de proficiencia en una o más competencias médicas, a partir del análisis de las necesidades sociales y de los pacientes ${ }^{1}$. Además, es un enfoque que enfatiza el entrenamiento basado en el tiempo a través de responsabilidad, flexibilidad y compromiso con la educación centrada en el estudiante ${ }^{1}$. Por lo tanto, para ser competente, es necesario el uso habitual y juicioso de diversos componentes como la comunicación, el conocimiento, las habilidades técnicas, el razonamiento clínico, las emociones, los valores y la reflexión, en la práctica diaria, en beneficio de la persona y la comunidad a la que se sirve ${ }^{2}$. Estos componentes cruciales para la practica, o dominios de competencia, contribuyen a la definición compresiva, holística e integral del ejercicio profesional.

A su vez, en el marco de la EBC, los dominios de competencia se describen como conjuntos de competencias mas pequeñas o especificas; esta ultimas son atributos o capacidades, duraderas y evaluables, que se pueden aprender, y que sirven para ejecutar una tarea, específica e integradora, 
dentro de una gama amplia de tareas que constituyen la profesión médica ${ }^{3}$. Así, por ejemplo, un dominio de competencia profesional es la comunicación médica, y sus competencias especificas son la empatía, la capacidad de escuchar, el lenguaje no verbal y la capacidad de escribir asertivamente.

Finalmente, las competencias y los dominios generales de competencia sirven para ejecutar tareas o responsabilidades específicas en ambientes de trabajo reales. Estas tareas o responsabilidades son denominadas Actividades Profesionales Confiables (APC). Las APC son unidades de práctica profesional que pueden ser confiadas a un aprendiz (por ejemplo, un estudiante o residente), una vez adquiere y domina las competencias específicas, que permiten ejecutar la actividad sin supervisión ${ }^{4}$. Por lo tanto, son unidades de práctica independientes, ejecutables dentro de un marco de tiempo, observables y evaluables en su proceso y resultado, y adecuadas para la toma de decisiones ${ }^{4}$.

La formación de cirujanos generales en el mundo no es ajena a esta transformación. La adopción de marcos de EBC en cirugía es, hoy en día, una realidad en diversos países europeos y norteamericanos ${ }^{5-8}$. Frente a los desafíos crecientes en materia de seguridad y cuidado del paciente, durante la ultima década, varias universidades y programas de cirugía han iniciado profundas transformaciones en sus currículos para responder a las expectativas de la sociedad. Estos cambios pretenden unificar y facilitar el diálogo académico entre programas, la transferencia de conocimiento, la evaluación de competencias profesionales y la adopción y garantía de estándares ajustados a niveles fijos de proficiencia quirúrgica entre los egresados.

En América Latina la experiencia con enfoques de EBC en cirugía es incipiente y aún se requieren esfuerzos para dar cuenta del tipo de cirujano que los países de la región pretenden formar, de acuerdo con las necesidades sociales, recursos y condiciones de práctica. Frente a este vacío, el objetivo del presente estudio fue desarrollar un consenso nacional sobre las mínimas competencias profesionales del cirujano egresado de los veinte programas de Especialización en Cirugía general en Colombia.

En este esfuerzo inicial, la División de Educación de la Asociación Colombiana de Cirugía (ACC), respetuosa de la autonomía universitaria y las políticas internas de cada uno de los programas de Especialización en Cirugía general, busca generar una primera aproximación sobre el perfil del cirujano que requiere el país. Igualmente se suma a las iniciativas en materia de talento humano propuestas por la Comisión para la transformación de la Educación Médica en Colombia, y a la Política Nacional de Talento Humano en Salud de la Dirección de Desarrollo del Talento Humano en Salud ${ }^{9,10}$.

\section{Materiales y métodos}

\section{Tipo de estudio}

\section{Consenso de expertos (método Delphi)}

El método Delphi pertenece al grupo de los métodos sistemáticos y estructurados para medir y desarrollar consenso, junto con la técnica de grupo nominal (TGN) y el método RAND (un mixto entre el Delphi y la TGN). Estos métodos son útiles cuando no existe evidencia empírica sobre un tema o cuando esta es limitada o contradictoria. Estas circunstancias son frecuentes en el campo de la Educación Médica en donde, además, el conocimiento es frecuentemente tácito y difícil de verbalizar y formalizar ${ }^{11}$.

El método Delphi consta de seis etapas: 1) identificar un problema de investigación; 2) realizar una búsqueda bibliográfica; 3) desarrollar un cuestionario de ítems para la evaluación por expertos; 4) realizar rondas iterativas anónimas para la evaluación de los ítems; 5) proporcionar retroalimentación individual y/o grupal entre rondas y; 6) sintetizar los hallazgos ${ }^{11,12}$. Este proceso se repite hasta alcanzar el mejor nivel posible de consenso o uno predeterminado que se puede completar en un número de rondas definidas.

Un aspecto importante es que los participantes (expertos) nunca se encuentran ni interactúan directamente. Por lo tanto, se pueden incluir un gran numero de participantes, obviando las limi- 
taciones geográficas, y se previene que algunos dominen la discusión y sesguen a otros participantes ${ }^{13}$.

A diferencia del Delphi, la TGN es presencial y, por lo general, involucra la interacción limitada entre cinco y doce participantes. Así, es posible discutir y debatir temas sin consenso. No obstante, involucra un número más pequeño de participantes y es difícil evitar que algunos de ellos dominen la discusión en el grupo. Por otra parte, el RAND es un método híbrido que comienza como un Delphi y luego finaliza con una TGN ${ }^{11}$. Sin embargo, presenta las limitaciones propias de la TGN.

\section{Identificación de fuentes primarias de información (búsqueda bibliográfica)}

A través de diferentes reuniones de la División de Educación de la ACC, los investigadores principales explicaron el propósito y alcance del consenso a los veinte directores de los programas de Especialización en Cirugía General del país. Inicialmente, la División de Educación solicitó el documento maestro del programa a cada uno de los directores. El documento maestro fue la fuente primaria de análisis en esta fase. Cada documento contiene la información detallada del programa (currículo, evaluación, estudiantes, profesores, entre otros), y esta unificado en sus criterios ante el Sistema Nacional de Educación Superior (SNIES) del Ministerio de Educación de Colombia.

Entre diciembre de 2019 y marzo de 2020, tres autores (LCD, AES y LT) realizaron el análisis documental de estas fuentes primarias de información. Mediante ese análisis se identificaron, organizaron, describieron y reportaron tres grupos principales de contenidos: 1) atributos profesionales generales que cada programa espera del residente durante su formación, 2) competencias prácticas (procedimientos quirúrgicos) que cada programa considera que los residentes deben realizar al final del entrenamiento y 3) Actividades Profesionales Confiables (APC) que los residentes deben ejecutar sin supervisión al final de su entrenamiento. Iterativamente LCD, AES y LT discutieron los contenidos principales de cada grupo hasta lograr consenso.

\section{Desarrollo de ítems del cuestionario}

Los ítems del instrumento fueron derivados a partir del análisis cualitativo de las fuentes primarias de información. En abril de 2020, LCD, AES y LT revisaron este listado de atributos profesionales generales, competencias prácticas (procedimientos quirúrgicos) y APC de manera independiente, y posteriormente compartieron sus percepciones hasta lograr un consenso sobre el contenido $\mathrm{y}$ aspectos formales de los ítems (por ejemplo, redacción, claridad, repetición). El cuestionario final fue desarrollado para conocer la perspectiva de los expertos sobre los atributos, competencias practicas (procedimientos) y APC que debe demostrar el residente de cirugía colombiano al final de su formación.

Para evaluar los atributos profesionales generales, el cuestionario cuenta con la siguiente instrucción para los participantes: "Desde su perspectiva, un residente de cirugía general colombiano debe demostrar los siguientes atributos", mediante una Escala de Likert (1: totalmente de acuerdo, 5: totalmente en desacuerdo). Algunos ejemplos de estos atributos son: "integrar eficientemente el conocimiento a la practica" y "ser honesto".

Para evaluar las competencias prácticas (procedimientos quirúrgicos), el cuestionario cuenta con la siguiente instrucción para los participantes: "Al final de su formación, un residente de cirugía colombiano debe ser competente para realizar los siguientes procedimientos de forma autónoma, independiente y sin supervisión. Por favor tenga en cuenta el perfil epidemiológico de su región, la disponibilidad de recursos disponibles (humanos y tecnológicos), la capacidad de su programa para ofrecer la posibilidad de un entrenamiento apropiado en estos procedimientos, los aspectos médico-legales, la posibilidad de remisión de pacientes a centros especializados en su ciudad o de otras ciudades, así como la experiencia acumulada por parte de los profesores en estos procedimientos quirúrgicos", mediante una Escala de Likert (1: totalmente de acuerdo, 5: totalmente en desacuerdo). Algunos ejemplos de estos procedimientos son: "apendicectomía abierta", "parotidectomia", "trasplante hepático". 
Para evaluar las APC, el cuestionario cuenta con la siguiente instrucción para los participantes: "Desde su perspectiva, un residente de cirugía general colombiano debe ser capaz de ejecutar estas actividades profesionales de forma confiable, autónoma, independiente y sin supervisión al final de su entrenamiento", mediante una Escala de Likert (1: totalmente de acuerdo, 5: totalmente en desacuerdo). Un ejemplo de las APC es: "Valorar pacientes quirúrgicos en el periodo perioperatorio".

\section{Rondas iterativas de evaluación: selección de participantes (expertos), proceso de respuesta y retroalimentación entre rondas}

En este consenso se consideraron como "expertos" a aquellos cirujanos que forman parte del cuerpo profesoral de cada uno de los programas de Especialización en Cirugía General del país. Para este fin, la División de Educación de la ACC, solicitó a los directores de programa el listado de profesores, independientemente de su experiencia, nivel de especialización y formación previa en educación medica. En total se identificaron 230 profesores.

Tras la prueba piloto del cuestionario con diez profesores de cirugía, con el fin de evaluar la claridad y redacción de los ítems, la versión final fue administrada en línea (Google Forms) a todos los expertos a través de los directores de programa. Para tal fin, se diseñaron tres rondas de evaluación de los ítems del cuestionario en los meses de mayo, junio y julio de 2020, respectivamente. Los expertos no recibieron ningún tipo de incentivo y su participación fue de forma voluntaria, confidencial y anónima. No se obtuvo consentimiento informado de los participantes. En el cuestionario se explicaron los propósitos del consenso, manejo y uso posterior de la información.

Durante la administración del cuestionario en la segunda y tercera rondas, se eliminaron aquellos ítems que presentaron alto consenso (mayor al 80\% entre los participantes) a favor o en contra. Igualmente, durante la segunda y tercera rondas se ofreció retroalimentación a los participantes sobre el resultado de aquellos ítems sin consenso en la ronda inmediatamente anterior.

\section{Síntesis de hallazgos: métodos y criterios de reporte del consenso}

Esta fase se realizó entre los meses de agosto y septiembre de 2020. Inicialmente se calcularon las estadísticas de las variables demográficas de los participantes (medias, desviaciones estándar y rangos). Al final de cada ronda, si más del $80 \%$ de los participantes concordaron en sus respuestas sobre cada atributo profesional, competencias prácticas (procedimiento quirúrgico) y APC, esta fue incluida o rechazada, según el caso. El "consenso a favor" se determinó con base en las respuestas "de acuerdo" o "totalmente de acuerdo" en la Escala de Likert. El consenso en contra se determinó con base en las respuestas "totalmente en desacuerdo", "en desacuerdo" o "neutro" en la Escala de Likert. Todos los ítems que presentaron concordancia inferior al $80 \%$ en la primera ronda fueron sometidos a una segunda ronda de evaluación, y así sucesivamente hasta completar tres rondas.

Al final de la tercera ronda de evaluación las respuestas definitivas fueron clasificadas en: 1) consenso a favor, 2) sin consenso o, 3) consenso en contra. Los atributos profesionales fueron agrupados por los investigadores en dominios generales de competencia profesional para facilitar su compresión por parte de la audiencia. Igualmente, los procedimientos quirúrgicos fueron agrupados en categorías generales de la cirugía de uso corriente (por ejemplo, cirugía vascular, torácica, gastrointestinal, trauma, etcétera).

Finalmente, los resultados del consenso y el manuscrito fueron revisados y aprobados por los directores de los programas de especialización. Igualmente, se adoptó un marco de referencia para el reporte de los resultados globales del consenso ${ }^{13}$.

\section{Resultados}

\section{Análisis de fuentes primarias y desarrollo del cuestionario}

Se revisó el 90 \% (n = 18) de los documentos maestro de los programas de Especialización en Cirugía General de Colombia registrados ante el SNIES. Todos los documentos revisados presentaron una descripción del perfil y los atributos 
profesionales esperados del residente durante su formación. Igualmente, todos contaron con una lista de procedimientos quirúrgicos (competencias practicas) en los que debe participar el residente durante su entrenamiento. Solo un documento describió las actividades profesionales confiables (APC) que debe realizar el residente al final de su entrenamiento, de forma autónoma, independiente y sin supervisión.

Luego de eliminar la información repetida y/o redundante entre los programas, se identificaron 100 atributos profesionales, 176 procedimientos quirúrgicos y 4 APC. Cada uno de los atributos, procedimientos y APC correspondieron a ítems individuales en el cuestionario final.

\section{Características demográficas de los participantes}

Un total de 105 profesores de cirugía, correspondientes al 45,6\% del cuerpo profesoral de los programas, participaron en las tres fases del consenso. El 14,3 \% de los participantes fueron mujeres. La edad promedio de los participantes fue 45,52 \pm 9,48 años (rango 29-70). El tiempo de experiencia de los cirujanos como profesores fue 11,5 $\pm 8,85$ años (rango 1-35).

La distribución de los cirujanos según la ubicación regional del programa, de acuerdo con la división vigente del Departamento Nacional de Estadística (DANE) ${ }^{14}$, fue: Bogotá D.C.: 58 cirujanos (55,2 \%); región Pacífica (departamentos de Cauca y Valle del Cauca): 13 cirujanos (12,4 \%); región Oriental (Santander y Cundinamarca): 8 cirujanos (7,6 \%); región Central (Caldas, Huila y Antioquia): 22 cirujanos (20,9\%); región Caribe (Bolívar): 4 cirujanos (3,8\%). Finalmente, 32 participantes $(30,5 \%)$ fueron profesores de programas públicos.

\section{Resultados de las tres rondas del consenso}

\section{Competencias profesionales generales}

En las dos primeras rondas de evaluación se obtuvo un consenso a favor ( $>80 \%$ ) en todas las competencias. No se identificó consenso en contra o competencias sin consenso. Estas competencias se agruparon en los siguientes dominios (Tabla 1):

Tabla 1. Dominios generales de competencias profesional para el egresado de los programas de Especialización en Cirugía General en Colombia

\begin{tabular}{|c|c|c|c|}
\hline \multicolumn{2}{|l|}{ Conocimiento } & \multirow{2}{*}{$\begin{array}{l}\text { Cuidado integral del paciente } \\
\text { Asumir complicaciones del ma- } \\
\text { nejo quirúrgico y brindar cuidado } \\
\text { continuo al paciente }\end{array}$} & \multirow{2}{*}{$\begin{array}{c}\text { Comunicación } \\
\text { Comunicarse asertiva, amable y } \\
\text { respetuosamente con el paciente }\end{array}$} \\
\hline Buscar y defender la verdad & $\begin{array}{l}\text { Ser analítico en la resolución } \\
\text { de problemas }\end{array}$ & & \\
\hline $\begin{array}{l}\text { Conducir investigación científi- } \\
\text { ca de alta calidad }\end{array}$ & Ser competitivo & $\begin{array}{l}\text { Atender integralmente a su pa- } \\
\text { ciente }\end{array}$ & $\begin{array}{l}\text { Comunicarse asertivamente (ver- } \\
\text { bal y escrita) con el publico en } \\
\text { general }\end{array}$ \\
\hline $\begin{array}{l}\text { Conocer el marco jurídico y } \\
\text { legal del ejercicio de la cirugía }\end{array}$ & Ser creativo & $\begin{array}{l}\text { Coordinar la atención de pa- } \\
\text { ciente y dirección del grupo de } \\
\text { trabajo involucrado }\end{array}$ & $\begin{array}{l}\text { Comunicarse asertivamente con } \\
\text { el grupo de trabajo }\end{array}$ \\
\hline $\begin{array}{l}\text { Conocer la carga de la enfer- } \\
\text { medad quirúrgica }\end{array}$ & Ser curioso & $\begin{array}{l}\text { Cuidar y seguir al paciente a lo } \\
\text { largo de toda la atención }\end{array}$ & $\begin{array}{l}\text { Comunicarse asertivamente en } \\
\text { presentaciones científicas (aca- } \\
\text { démicas) }\end{array}$ \\
\hline $\begin{array}{l}\text { Conocer la epidemiologia y } \\
\text { demografía de la enfermedad } \\
\text { quirúrgica }\end{array}$ & Ser emprendedor & $\begin{array}{l}\text { Diligenciar adecuadamente el } \\
\text { consentimiento informado de } \\
\text { todos los procedimientos que } \\
\text { realiza }\end{array}$ & $\begin{array}{l}\text { Demostrar amabilidad en sus re- } \\
\text { laciones interpersonales }\end{array}$ \\
\hline $\begin{array}{l}\text { Conocer los fundamentos cien- } \\
\text { tíficos de la cirugía (ciencias } \\
\text { básicas, clínicas) }\end{array}$ & Ser innovador & $\begin{array}{l}\text { Diligenciar apropiada y comple- } \\
\text { tamente todos los aspectos de } \\
\text { la historia clínica }\end{array}$ & Demostrar calidad humana \\
\hline $\begin{array}{l}\text { Argumentar sus puntos de vista } \\
\text { coherentemente }\end{array}$ & $\begin{array}{l}\text { Supervisar a otro personal en } \\
\text { formación }\end{array}$ & Educar al paciente y familia & Escuchar a los demás \\
\hline Demostrar rigor científico & $\begin{array}{l}\text { Tener conocimientos genera- } \\
\text { les (historia, política, cultura } \\
\text { etc.). }\end{array}$ & $\begin{array}{l}\text { Gestionar el riesgo individual del } \\
\text { paciente (riesgo/beneficio de las } \\
\text { intervenciones que realiza) }\end{array}$ & $\begin{array}{l}\text { Negociar y solucionar apropiada- } \\
\text { mente los conflictos }\end{array}$ \\
\hline
\end{tabular}


Continuación tabla 1

\begin{tabular}{|c|c|c|c|}
\hline \multicolumn{2}{|l|}{ Conocimiento } & \multirow{2}{*}{$\begin{array}{l}\text { Cuidado integral del paciente } \\
\text { Prevenir la enfermedad y promo- } \\
\text { ver activamente la salud }\end{array}$} & \multirow{2}{*}{\begin{tabular}{l}
\multicolumn{1}{|c|}{ Comunicación } \\
Reportar oportunamente los erro \\
res médicos y aportar soluciones \\
constructivas
\end{tabular}} \\
\hline $\begin{array}{l}\text { Educar a otros profesionales } \\
\text { (ensenar) }\end{array}$ & $\begin{array}{l}\text { Tener destreza técnica ope- } \\
\text { ratoria }\end{array}$ & & \\
\hline $\begin{array}{l}\text { Integrar eficientemente el cono- } \\
\text { cimiento a la practica }\end{array}$ & $\begin{array}{l}\text { Tener habilidades clínicas } \\
\text { (diagnostico) }\end{array}$ & $\begin{array}{l}\text { Respetar la privacidad, dignidad, } \\
\text { libertad y autonomía del paciente }\end{array}$ & $\begin{array}{l}\text { Ser competente y fluido en una } \\
\text { segunda lengua (hablar, escribir, } \\
\text { leer) }\end{array}$ \\
\hline $\begin{array}{l}\text { Leer y analizar críticamente la } \\
\text { información y evidencia cientí- } \\
\text { fica }\end{array}$ & $\begin{array}{l}\text { Tener habilidades clínicas de } \\
\text { tratamiento y manejo perio- } \\
\text { peratorio }\end{array}$ & $\begin{array}{l}\text { Ser compasivo ante el sufrimiento } \\
\text { del paciente }\end{array}$ & $\begin{array}{l}\text { Utilizar apropiadamente las tecno- } \\
\text { logías digitales y de información } \\
\text { disponibles. }\end{array}$ \\
\hline Pensar críticamente & $\begin{array}{l}\text { Tener habilidades clínicas } \\
\text { para la obtener información } \\
\text { relevante del paciente }\end{array}$ & $\begin{array}{l}\text { Ser proactivo en el cuidado del } \\
\text { paciente }\end{array}$ & \\
\hline $\begin{array}{l}\text { Trasladar apropiadamente la } \\
\text { teoría quirúrgica y evidencia } \\
\text { científica a la practica }\end{array}$ & Tener juicio clínico & $\begin{array}{l}\text { Liderar la atención de pacientes } \\
\text { a su cargo }\end{array}$ & \\
\hline \multirow[t]{3}{*}{$\begin{array}{l}\text { Usar apropiadamente la medici- } \\
\text { na basada en evidencia }\end{array}$} & & $\begin{array}{l}\text { Tomar decisiones acertadas de } \\
\text { acuerdo con la condición indivi- } \\
\text { dual del paciente y recursos dis- } \\
\text { ponibles. }\end{array}$ & \\
\hline & & Trabajar en equipo & \\
\hline & & $\begin{array}{l}\text { Trabajar interprofesional e inter- } \\
\text { disciplinarmente }\end{array}$ & \\
\hline \multicolumn{2}{|l|}{ Ética } & \multicolumn{2}{|c|}{ Practica basada en el sistema de salud } \\
\hline Aprender toda la vida. & $\begin{array}{l}\text { Respetar a sus compañeros, } \\
\text { profesores y directivos }\end{array}$ & \multicolumn{2}{|c|}{$\begin{array}{l}\text { Abogar por la atención de calidad para el paciente dentro del sistema } \\
\text { sanitario }\end{array}$} \\
\hline Autoevaluarse permanente & Respetar la vida & \multicolumn{2}{|c|}{ Actuar como un agente de cambio positivo en su entorno } \\
\hline Autorregularse & $\begin{array}{l}\text { Respetar las diferencias y dig- } \\
\text { nidad de los demás }\end{array}$ & \multicolumn{2}{|c|}{$\begin{array}{l}\text { Adaptarse al ejercicio en diversos contextos de practica (urgencias, } \\
\text { hospitalización, sector publico/privado) }\end{array}$} \\
\hline Brindar ayuda a los demás & $\begin{array}{l}\text { Respetar y considerar a sus } \\
\text { colegas }\end{array}$ & \multicolumn{2}{|c|}{$\begin{array}{l}\text { Adaptarse y cumplir con las normas institucionales (donde se } \\
\text { encuentre trabajando) }\end{array}$} \\
\hline $\begin{array}{l}\text { Buscar ayuda y reconocer sus } \\
\text { propios limites }\end{array}$ & Ser auto determinado & \multicolumn{2}{|c|}{$\begin{array}{l}\text { Administrar adecuadamente los recursos tecnológico, económico y } \\
\text { humano que tenga a su cargo }\end{array}$} \\
\hline $\begin{array}{l}\text { Comprender el sufrimiento de } \\
\text { los demás }\end{array}$ & Ser autónomo & \multicolumn{2}{|c|}{$\begin{array}{l}\text { Comprometerse con el funcionamiento ágil y eficiente del servicio } \\
\text { donde se encuentre trabajando }\end{array}$} \\
\hline $\begin{array}{l}\text { Comprometerse con la respon- } \\
\text { sabilidad legal de sus acciones }\end{array}$ & Ser confiable & \multicolumn{2}{|c|}{ Comprometerse socialmente para el mejoramiento del país } \\
\hline $\begin{array}{l}\text { Conocer los principios de la éti- } \\
\text { ca medica }\end{array}$ & Ser honesto & \multicolumn{2}{|c|}{ Conocer el sistema de salud y principios de calidad } \\
\hline Demostrar colegaje & Ser idóneo & \multicolumn{2}{|c|}{ Cooperar y trabajar con aseguradores y auditores } \\
\hline $\begin{array}{l}\text { Demostrar gratitud hacia sus } \\
\text { profesores y universidad }\end{array}$ & Ser integro & \multicolumn{2}{|c|}{ Demostrar costo conciencia en el uso de recursos } \\
\hline Demostrar humanismo & $\begin{array}{l}\text { Ser prudente en las decisiones } \\
\text { clínicas }\end{array}$ & \multicolumn{2}{|c|}{$\begin{array}{l}\text { Diligenciar adecuadamente las solicitudes administrativas y ordenes } \\
\text { de atención de sus pacientes }\end{array}$} \\
\hline $\begin{array}{l}\text { Demostrar responsabilidad en } \\
\text { su actuar profesional }\end{array}$ & $\begin{array}{l}\text { Ser puntual en sus obligacio- } \\
\text { nes }\end{array}$ & \multicolumn{2}{|c|}{ Reconocer y apropiar los principios de calidad y seguridad del paciente } \\
\hline $\begin{array}{l}\text { Demostrar sensibilidad hacia } \\
\text { poblaciones diversas (genero, } \\
\text { raza, cultura) }\end{array}$ & Ser transparente & \multicolumn{2}{|l|}{ Ser coherente en el uso de recursos } \\
\hline $\begin{array}{l}\text { Pensar en los demás por enci- } \\
\text { ma de si mismo }\end{array}$ & Ser un buen ciudadano & \multicolumn{2}{|l|}{ Ser flexible al cambio organizacional } \\
\hline $\begin{array}{l}\text { Planificar y organizar su propio } \\
\text { trabajo }\end{array}$ & $\begin{array}{l}\text { Equilibrar apropiadamente la } \\
\text { vida laboral, personal y fami- } \\
\text { liar. }\end{array}$ & \multicolumn{2}{|l|}{ Servir a la comunidad } \\
\hline $\begin{array}{l}\text { Reflexionar permanente sobre } \\
\text { su propio desempeño }\end{array}$ & & & \\
\hline
\end{tabular}

* Todas las competencias de cada dominio demostraron ALTO consenso entre los participantes del consenso (> 80 \%) durante la primera y segunda ronda del método Delphi 
a. Conocimiento quirúrgico

b. Comunicación

c. Ética

d. Cuidado integral del paciente

e. Practica basada en el sistema de salud

\section{Competencias practicas (procedimientos quirúrgicos)}

En las tres rondas de evaluación se obtuvo consenso a favor (> $80 \%$ ) en un $66,5 \%$ de los procedimientos $(\mathrm{n}=117)$ (Tabla 2). Durante las tres rondas se obtuvo consenso en contra (> $80 \%$ ) en $6,8 \%$ de los procedimientos $(n=12)$ (Tabla 3$)$. No se obtuvo consenso en $26,7 \%$ de los procedimientos quirúrgicos $(\mathrm{n}=47)$ (Tabla 4).
3. Actividades Profesionales Confiables

En la primera ronda de evaluación se obtuvo consenso a favor (> $80 \%$ ) en todas las APC identificadas, que fueron:

a. Valorar adultos con condiciones quirúrgicas urgentes de causa médica y traumática en servicios de urgencia y hospitalarios.

b. Valorar adultos con condiciones quirúrgicas electivas en servicios de consulta externa y servicios ambulatorios.

c. Realizar procedimientos quirúrgicos específicos.

d. Valorar pacientes quirúrgicos en el periodo perioperatorio.

Tabla 2. Competencias practicas (procedimientos quirúrgicos) con ALTO CONSENSO a FAVOR entre los participantes.

\begin{tabular}{|c|c|c|c|c|}
\hline \multirow{2}{*}{ Área } & \multirow{2}{*}{ Procedimiento } & \multicolumn{3}{|c|}{$\begin{array}{l}\text { Porcentaje de acuerdo entre } \\
\text { los participantes* }\end{array}$} \\
\hline & & $\begin{array}{l}\text { Primera } \\
\text { ronda }\end{array}$ & $\begin{array}{l}\text { Segunda } \\
\text { ronda }\end{array}$ & $\begin{array}{c}\text { Tercera } \\
\text { ronda }\end{array}$ \\
\hline \multirow{4}{*}{ Cabeza y cuello } & Tiroidectomía subtotal por vía abierta & 69,5 & 81,9 & \\
\hline & Tiroidectomía total por vía abierta & 67,1 & 81,9 & \\
\hline & Traqueostomía percutánea & 91,4 & & \\
\hline & Traquestomia abierta & 100 & & \\
\hline \multirow{22}{*}{ Gastrointestinal } & Gastrectomía total por vía abierta & 86,6 & & \\
\hline & Corrección de hernia hiatal (deslizamiento/ paraesofágica) por vía abierta & 69,5 & 78,1 & 85,7 \\
\hline & Gastrectomía subtotal abierta con reconstrucción en Y de Roux o Bll & 90,2 & & \\
\hline & Gastrectomía parcial vía abierta & 93,9 & & \\
\hline & Gastroyeyunostomía abierta & 98,7 & & \\
\hline & Gastroyeyunostomía laparoscópica & 59,7 & 66,7 & 80 \\
\hline & Piloroplastia vía abierta & 97,6 & & \\
\hline & Vagotomía troncular vía abierta & 89,0 & & \\
\hline & $\begin{array}{l}\text { Manejo quirúrgico avanzado de una perforación duodenal (exclusión } \\
\text { pilórica, derivación biliar, parche de serosa) por vía abierta }\end{array}$ & 95,1 & & \\
\hline & $\begin{array}{l}\text { Manejo quirúrgico avanzado de una perforación esofágica (esofagec- } \\
\text { tomía parcial, avance de parches, exclusión esofágica) por vía abierta } \\
\text { en } 1,2 \text { o } 3 \text { vías }\end{array}$ & 84,1 & & \\
\hline & $\begin{array}{l}\text { Manejo quirúrgico de una perforación duodenal (sutura duodenal, lavado) } \\
\text { por vía abierta }\end{array}$ & 100 & & \\
\hline & $\begin{array}{l}\text { Manejo quirúrgico de una perforación duodenal (sutura duodenal, lavado) } \\
\text { por vía laparoscópica }\end{array}$ & 54,9 & 86,7 & \\
\hline & Sutura de ulcera péptica sangrante por vía abierta & 98,8 & & \\
\hline & Avance de sonda de nutrición enteral & 87,8 & & \\
\hline & Gastrostomía para drenaje y/o alimentación por vía abierta & 100 & & \\
\hline & Gastrostomía para drenaje y/o alimentación por vía laparoscópica & 78,1 & 88,6 & \\
\hline & Yeyunostomia de alimentación por vía abierta & 98,8 & & \\
\hline & Anastomosis de intestino delgado por vía abierta & 100 & & \\
\hline & Anastomosis de intestino delgado por vía laparoscópica & 65,8 & 80 & \\
\hline & Biopsia de órganos solidos intraperitoneal (hígado) por vía abierta & 96,3 & & \\
\hline & Esplenectomía abierta & 97,6 & & \\
\hline & Esplenectomía laparoscópica & 71,9 & 82,9 & \\
\hline
\end{tabular}


Continuación tabla 2

\begin{tabular}{|c|c|c|c|c|}
\hline \multirow{2}{*}{ Área } & \multirow{2}{*}{ Procedimiento } & \multicolumn{3}{|c|}{$\begin{array}{l}\text { Porcentaje de acuerdo entre los } \\
\text { participantes* }\end{array}$} \\
\hline & & $\begin{array}{l}\text { Primera } \\
\text { ronda }\end{array}$ & $\begin{array}{l}\text { Segunda } \\
\text { ronda }\end{array}$ & $\begin{array}{l}\text { Tercera } \\
\text { ronda }\end{array}$ \\
\hline \multirow{2}{*}{ Apéndice cecal } & Apendicectomía abierta & 100 & & \\
\hline & Apendicectomía laparoscópica & 97,6 & & \\
\hline \multirow{21}{*}{$\begin{array}{l}\text { Pared abdominal } \\
\text { y peritoneo }\end{array}$} & Drenaje colección intrabdominal por laparoscopia & 96,3 & & \\
\hline & Drenaje colección intrabdominal por laparotomía & 100 & & \\
\hline & Empaquetamiento abdominal & 100 & & \\
\hline & Herniorrafia inguinal abierta (anterior y pre peritoneal) & 100 & & \\
\hline & Herniorrafia inguinal laparoscópica (TAPP, TEP) & 82,9 & & \\
\hline & Herniorrafia umbilical y epigástrica por vía abierta & 100 & & \\
\hline & Herniorrafia umbilical y epigástrica por vía laparoscópica & 69,5 & 80 & \\
\hline & Inserción y/o retiro de un catéter para diálisis peritoneal por vía abierta & 93,9 & & \\
\hline & Laparoscopia diagnostica & 100 & & \\
\hline & Lavado peritoneal terapéutico en abdomen abierto & 100 & & \\
\hline & Colocación se sistema de presión negativa en abdomen abierto & 100 & & \\
\hline & Liberación de adherencias peritoneales por vía abierta & 100 & & \\
\hline & Liberación de adherencias peritoneales por vía laparoscópica & 85,4 & & \\
\hline & Lavado peritoneal diagnostico & 85,4 & & \\
\hline & $\begin{array}{l}\text { Reparo de una hernia compleja de la pared abdominal (ventral) por } \\
\text { vía abierta. }\end{array}$ & 91,5 & & \\
\hline & Reparo de una hernia para-ostomal por vía abierta & 95,1 & & \\
\hline & $\begin{array}{l}\text { Reparo de una hernia reproducida (inguinal, umbilical y/o epigástrica) } \\
\text { por vía abierta }\end{array}$ & 100 & & \\
\hline & $\begin{array}{l}\text { Reparo de una hernia reproducida (inguinal, umbilical y/o epigástrica) } \\
\text { por vía laparoscópica }\end{array}$ & 62,2 & 82,9 & \\
\hline & Biopsia de peritoneo vía abierta & 100 & & \\
\hline & Biopsia de peritoneo vía laparoscópica & 97,6 & & \\
\hline & Curación de una herida quirúrgica. & 98,8 & & \\
\hline \multirow{8}{*}{$\begin{array}{l}\text { Piel y tejidos } \\
\text { blandos }\end{array}$} & Drenaje de abscesos superficiales & 100 & & \\
\hline & Drenaje de absceso pilonidal & 100 & & \\
\hline & Resección de quiste pilonidal & 96,3 & & \\
\hline & Resección de lesiones benignas de la piel y TCS & 100 & & \\
\hline & Desbridamiento de tejidos en infecciones necrotizantes & 100 & & \\
\hline & Biopsia de piel y musculo & 100 & & \\
\hline & Biopsia de ganglio inguinal, axilar o cervical profundo & 98,8 & & \\
\hline & Biopsia de ganglio inguinal, axilar o cervical superficial & 98,8 & & \\
\hline \multirow{3}{*}{ Mama } & Biopsia abierta de tejido mamario sospechoso de malignidad & 84,1 & & \\
\hline & Drenaje de absceso mamario & 95,1 & & \\
\hline & Resección de lesión benigna en glándula mamaria (fibroadenoma). & 75,6 & 87,6 & \\
\hline
\end{tabular}


Continuación tabla 2

\begin{tabular}{|c|c|c|c|c|}
\hline \multirow{2}{*}{ Área } & \multirow{2}{*}{ Procedimiento } & \multicolumn{3}{|c|}{$\begin{array}{l}\text { Porcentaje de acuerdo entre } \\
\text { los participantes* }\end{array}$} \\
\hline & & $\begin{array}{l}\text { Primera } \\
\text { ronda }\end{array}$ & $\begin{array}{l}\text { Segunda } \\
\text { ronda }\end{array}$ & $\begin{array}{c}\text { Tercera } \\
\text { ronda }\end{array}$ \\
\hline \multirow{15}{*}{$\begin{array}{l}\text { Colon, recto } \\
\text { y ano }\end{array}$} & Anastomosis colo-colonica por vía abierta & 100 & & \\
\hline & Cierre de un muñón rectal por vía abierta & 98,8 & & \\
\hline & Cierre de una colostomía y/o ileostomía en asa por vía abierta & 98,8 & & \\
\hline & Colectomia derecha abierta & 98,8 & & \\
\hline & Colectomia izquierda abierta & 97,6 & & \\
\hline & Colectomia total por vía abierta & 91,5 & & \\
\hline & Resección anterior de recto vía abierta & 70,7 & 73,3 & 85,7 \\
\hline & Colostomía y/o ileostomía asistida por vía laparoscópica & 71,9 & 81,9 & \\
\hline & Colostomía y/o ileostomía por vía abierta. & 100 & & \\
\hline & Drenaje de absceso ano-rectal & 97,6 & & \\
\hline & Drenaje de hemorroides trombosadas & 98,8 & & \\
\hline & Fistulotomia anal en casos de fistulas simples & 79,3 & 84,8 & \\
\hline & Hemorroidectomia por técnica abierta convencional & 87,8 & & \\
\hline & Sigmoidectomia y cierre distal del muñón rectal (Hartmann) por vía abierta & 96,3 & & \\
\hline & Retiro de cuerpo extraño rectal & 95,1 & & \\
\hline \multirow{6}{*}{ Tórax } & Corrección de hernia diafragmática por vía abierta & 76,8 & 81,9 & \\
\hline & Decorticacion pleural por vía abierta & 85,4 & & \\
\hline & Toracentesis evacuatoria & 100 & & \\
\hline & Toracostomía drenaje cerrado & 100 & & \\
\hline & Biopsia pleural vía abierta & 89,0 & & \\
\hline & Biopsia pleural vía toracoscopica & 78,1 & 81,9 & \\
\hline \multirow{18}{*}{ Trauma } & Cardiorafia & 100 & & \\
\hline & Ecografía de pericardio y FAST & 89,0 & & \\
\hline & Esternotomia & 100 & & \\
\hline & Exploración y reparación de grandes vasos en el abdomen & 100 & & \\
\hline & Exploración vascular o aero-digestiva del cuello & 98,8 & & \\
\hline & Exploración y reconstrucción vascular en extremidades & 96,3 & & \\
\hline & Fasciotomia & 97,6 & & \\
\hline & Sutura de grandes vasos torácicos & 100 & & \\
\hline & Sutura de pulmón / lobectomía / neumonectomia & 97,6 & & \\
\hline & Sutura de pulmón por vía abierta & 96,3 & & \\
\hline & Sutura hepática por laparotomía & 100 & & \\
\hline & Sutura hepática por laparoscopia & 59,8 & 72,4 & 80 \\
\hline & Toracotomía de resucitación & 98,8 & & \\
\hline & Ventana pericárdica & 98,8 & & \\
\hline & Cricotiroidotomia abierta y/o aguja & 96,3 & & \\
\hline & Sutura de tráquea & 98,8 & & \\
\hline & Sutura de diafragma & 100 & & \\
\hline & Sutura de vasos de la pared torácica & 98,8 & & \\
\hline
\end{tabular}


Continuación tabla 2

\begin{tabular}{|c|c|c|c|c|}
\hline \multirow{2}{*}{ Área } & \multirow{2}{*}{ Procedimiento } & \multicolumn{3}{|c|}{$\begin{array}{l}\text { Porcentaje de acuerdo entre } \\
\text { los participantes* }\end{array}$} \\
\hline & & $\begin{array}{l}\text { Primera } \\
\text { ronda }\end{array}$ & $\begin{array}{l}\text { Segunda } \\
\text { ronda }\end{array}$ & $\begin{array}{c}\text { Tercera } \\
\text { ronda }\end{array}$ \\
\hline \multirow{5}{*}{ Vascular } & Inserción de catéter venoso central implantable. & 95,1 & & \\
\hline & $\begin{array}{l}\text { Exploración vascular de un paciente con isquemia critica de la extremidad } \\
\text { inferior }\end{array}$ & 54,9 & 69,5 & 81,9 \\
\hline & Inserción de un catéter central no implantable & 98,8 & & \\
\hline & Varicosafenectomia & 78,1 & 89,6 & \\
\hline & Venodiseccion & 91,5 & & \\
\hline \multirow{12}{*}{$\begin{array}{l}\text { Hepato bilio } \\
\text { pancreatico }\end{array}$} & Esfinteroplastia transduodenal & 70,7 & 80,9 & \\
\hline & Colecistectomía abierta & 98,8 & & \\
\hline & Colecistectomía laparoscópica & 100 & & \\
\hline & Drenaje de absceso hepático por vía abierta y/o laparoscópica & 100 & & \\
\hline & Exploración de vía biliar por vía abierta & 97,6 & & \\
\hline & Exploración de vía biliar por vía laparoscópica & 61,0 & 75,2 & 80 \\
\hline & Marsupializacion de la celda pancreática. & 81,7 & & \\
\hline & Necrosectomia pancreática por vía abierta & 97,6 & & \\
\hline & Pancreatectomia distal por vía abierta & 80,5 & & \\
\hline & Drenaje de pseudoquiste pancreático vía abierta & 78,1 & 86,7 & \\
\hline & Destechamiento de quiste hepático por vía abierta & 85,4 & & \\
\hline & Destechamiento de quiste hepático por vía laparoscópica & 69,5 & 88,6 & \\
\hline Pediátrica & Reparo abierto de una hernia inguinal, epigástrica y umbilical en un niño & 69,5 & 73,3 & 86,7 \\
\hline
\end{tabular}

Este listado denota las competencias practicas que debe demostrar un egresado de los programas de Especialización en Cirugía General en Colombia, de forma autónoma, independiente y sin supervisión.

* Significativo si > $80 \%$

Tabla 3. Competencias practicas (procedimientos quirúrgicos) con ALTO CONSENSO en CONTRA entre los participantes.

\begin{tabular}{|l|l|c|}
\hline \multirow{4}{*}{\multicolumn{1}{|c|}{ Área }} & \multicolumn{1}{|c|}{ Procedimiento } & $\begin{array}{c}\text { Porcentaje de acuerdo entre } \\
\text { los participantes* }\end{array}$ \\
\cline { 3 - 3 } & & Primera ronda \\
\hline \multirow{4}{*}{ Cabeza y cuello } & Laringuectomía & 95,1 \\
\cline { 2 - 3 } & Paratiroidectomía & 87,8 \\
\cline { 2 - 3 } & Parotidectomía & 86,6 \\
\cline { 2 - 3 } & COMANDO & 97,6 \\
\hline Gastrointestinal & Baipás gástrico para obesidad & 89,0 \\
\hline \multirow{2}{*}{ Trasplantes } & Trasplante hepático & 100 \\
\cline { 2 - 3 } & Trasplante renal & 98,8 \\
\hline \multirow{2}{*}{ Hepatobiliopancreatico } & Shunt porto sistémico para HTP & 97,6 \\
\cline { 2 - 3 } & CPRE & 97,6 \\
\hline Tórax & Resección tumor mediastinal & 92,7 \\
\hline \multirow{2}{*}{ Vascular } & Reparo electivo de un aneurisma de aorta abdominal & 87,8 \\
\cline { 2 - 3 } & Endarterectomia carotidea & 91,5 \\
\hline
\end{tabular}


Tabla 4. Competencias practicas (procedimientos quirúrgicos) SIN CONSENSO entre los participantes

\begin{tabular}{|c|c|c|c|c|}
\hline \multirow{2}{*}{ Área } & \multirow{2}{*}{ Procedimiento } & \multicolumn{3}{|c|}{$\begin{array}{l}\text { Porcentaje de acuerdo entre } \\
\text { los participantes* }\end{array}$} \\
\hline & & $\begin{array}{c}\text { Primera } \\
\text { ronda }\end{array}$ & $\begin{array}{l}\text { Segunda } \\
\text { ronda }\end{array}$ & $\begin{array}{c}\text { Tercera } \\
\text { ronda }\end{array}$ \\
\hline \multirow{13}{*}{ Gastrointestinal } & Adrenalectomía abierta & 53,7 & 56,2 & 60,9 \\
\hline & Adrenalectomía laparoscópica & 25,6 & 33,3 & 37,1 \\
\hline & Endoscopia digestiva alta con fines diagnósticos & 30,5 & 39,1 & 29,5 \\
\hline & Esofagectomía abierta por vía transhiatal, torácica o tres vías. & 52,4 & 65,7 & 63,8 \\
\hline & Gastrectomía total por vía laparoscópica & 29,3 & 35,2 & 24,8 \\
\hline & Cirugía antirreflejo vía laparoscópica & 53,7 & 64,8 & 62,9 \\
\hline & Cirugía antirreflejo vía abierta & 63,4 & 74,3 & 74,3 \\
\hline & $\begin{array}{l}\text { Corrección de hernia hiatal (deslizamiento/ para esofágica) } \\
\text { por vía laparoscópica }\end{array}$ & 46,3 & 61,0 & 59,1 \\
\hline & Gastrectomía subtotal laparoscópica con reconstrucción B II & 45,1 & 47,6 & 43,8 \\
\hline & Gastrectomía parcial vía laparoscópica & 45,1 & 55,2 & 50,5 \\
\hline & Piloroplastia vía laparoscópica & 42,7 & 57,1 & 61,0 \\
\hline & Vagotomía troncular vía laparoscópica & 52,4 & 56,2 & 57,1 \\
\hline & $\begin{array}{l}\text { Reconstrucción compleja del tracto digestivo (ascenso gás- } \\
\text { trico, ascenso colonico) por vía abierta }\end{array}$ & 45,1 & 46,7 & 48,6 \\
\hline \multirow{15}{*}{ Colon, recto y ano } & Anastomosis colo-colonica por vía laparoscópica & 48,8 & 44,8 & 49,5 \\
\hline & Cierre de un muñón rectal por vía laparoscópica & 47,6 & 52,4 & 50,5 \\
\hline & Colectomía derecha laparoscópica & 62,2 & 67,6 & 61,0 \\
\hline & Colectomía izquierda laparoscópica & 51,2 & 42,9 & 42,9 \\
\hline & Colectomía total por vía laparoscópica & 32,9 & 24,8 & 19,1 \\
\hline & Resección anterior de recto vía laparoscópica & 23,2 & 19,1 & 21,0 \\
\hline & Resección anterior baja de recto vía abierta & 62,2 & 73,3 & 71,4 \\
\hline & Resección anterior baja de recto vía laparoscópica & 19,5 & 61,9 & 18,1 \\
\hline & Resección abdomino perineal & 40,2 & 16,2 & 45,7 \\
\hline & $\begin{array}{l}\text { Colocación de un seton cortante en casos de fistulas ano-rec- } \\
\text { tales complejas }\end{array}$ & 62,2 & 73,3 & 78,1 \\
\hline & Colonoscopia total con fines diagnósticos & 28,1 & 28,6 & 22,9 \\
\hline & Rectoscopia con fines diagnósticos. & 68,3 & 65,7 & 63,8 \\
\hline & Esfinterotomia anal lateral & 59,8 & 70,5 & 74,3 \\
\hline & Corrección de prolapso rectal & 32,9 & 41,0 & 38,1 \\
\hline & Corrección de incontinencia fecal. & 11,0 & 16,2 & 17,0 \\
\hline \multirow{2}{*}{$\begin{array}{l}\text { Piel y tejidos } \\
\text { blandos }\end{array}$} & Resección de tumores retroperitoneales vía abierta & 42,7 & 50,5 & 61,0 \\
\hline & $\begin{array}{l}\text { Resección de tumores malignos (sarcoma, melanoma) en } \\
\text { extremidades }\end{array}$ & 29,3 & 22,7 & 19,1 \\
\hline
\end{tabular}




\begin{tabular}{|c|c|c|c|c|}
\hline \multirow{2}{*}{ Área } & \multirow{2}{*}{ Procedimiento } & \multicolumn{3}{|c|}{$\begin{array}{l}\text { Porcentaje de acuerdo entre } \\
\text { los participantes* }\end{array}$} \\
\hline & & $\begin{array}{l}\text { Primera } \\
\text { ronda }\end{array}$ & $\begin{array}{l}\text { Segunda } \\
\text { ronda }\end{array}$ & $\begin{array}{l}\text { Tercera } \\
\text { ronda }\end{array}$ \\
\hline \multirow{6}{*}{$\begin{array}{l}\text { Hepatobilio } \\
\text { pancreático }\end{array}$} & Hepatectomía abierta & 31,7 & 45,7 & 37,1 \\
\hline & Hepaticoyeyunostomía abierta & 58,5 & 68,6 & 77,1 \\
\hline & Necrosectomía pancreática por vía laparoscópica & 48,8 & 70,5 & 71,4 \\
\hline & Pancreatectomía distal por vía laparoscópica & 32,9 & 46,7 & 51,4 \\
\hline & Pancreato-duodenectomía abierta & 40,2 & 41,0 & 36,2 \\
\hline & Drenaje de pseudoquiste pancreático vía laparoscópica & 41,5 & 72,4 & 68,6 \\
\hline \multirow{2}{*}{ Mama } & Mastectomía radical modificada & 18,3 & 40,0 & 38,1 \\
\hline & Mastectomía simple & 41,5 & 60,0 & 71,4 \\
\hline Pediátrica & Exploración escrotal por torsión testicular y/o criptorquidia & 32,9 & 34,3 & 46,7 \\
\hline \multirow{6}{*}{ Tórax } & Biopsia toracoscopica de lesión en mediastino & 17,1 & 30,5 & 27,6 \\
\hline & Biopsia abierta de lesión en mediastino & 39,0 & 37,1 & 38,1 \\
\hline & Biopsia de pulmón por vía abierta & 59,8 & 70,5 & 75,2 \\
\hline & Biopsia de pulmón por vía toracoscópica & 35,4 & 66,8 & 66,8 \\
\hline & $\begin{array}{l}\text { Corrección de hernia diafragmática por vía laparoscópica } \\
\text { y/o toracoscópica. }\end{array}$ & 32,9 & 50,5 & 60,0 \\
\hline & Decorticación pleural por vía toracoscópica & 51,2 & 78,1 & 78,1 \\
\hline \multirow{2}{*}{ Vascular } & Amputación de extremidades (superior / inferior) & 70,7 & 74,3 & 75,2 \\
\hline & Reparo de un aneurisma de aorta abdominal roto & 61,0 & 74,3 & 78,1 \\
\hline
\end{tabular}

* Significativo si $>80 \%$

\section{Discusión}

El objetivo de este estudio fue realizar un consenso sobre los atributos profesionales que debe demostrar un residente de cirugía colombiano, las competencias prácticas (procedimientos quirúrgicos) y las Actividades Profesionales Confiables (APC) que debe ejecutar al final de su formación (autónoma, independientemente y sin supervisión). Los resultados principales demostraron alto consenso por parte de los profesores de los veinte programas de Especialización en Cirugía General, en el $100 \%$ de los atributos profesionales declarados por cada programa en el documento maestro registrado ante el SNIES del Ministerio de Educación Nacional. El consenso agrupó estos atributos en cinco dominios generales de competencia profesional, estrechamente relacionados, para facilitar la compresión de la formación integral del residente: conocimiento, comunicación, ética, cuidado integral del paciente y práctica basada en el sistema de salud.

Un segundo hallazgo indicó alto consenso a favor en el 66,5 \% de las competencias prácticas declaradas por los programas (117 procedimientos quirúrgicos) y alto consenso en contra en el 6,8\% las competencias prácticas (12 procedimientos). El estudio no informó consenso en $26,7 \%$ de las competencias prácticas restantes (correspondientes a 47 procedimientos quirúrgicos). Finalmente, se obtuvo alto consenso en cuatro APC que deben realizar los residentes al final de su formación.

El primer hallazgo resalta la importancia de la formación integral del cirujano en múltiples dominios de competencia y no solo en el entrenamiento operatorio. La definición de dominios de competencia explícitos contribuye a que las profesiones sanitarias estén bien definidas, a 
promover una fuerza laboral competente, a facilitar la evaluación y la movilidad profesional, y al análisis y evaluación de la profesión ${ }^{15}$. Por lo tanto, este consenso aporta valiosa información con respecto a las cualidades ideales del cirujano colombiano, teniendo en cuenta la dinámica de la profesión en el país, el perfil epidemiológico y demográfico, y las características de la fuerza y el mercado laboral. En este sentido, los resultados concuerdan con la tendencia global que enfatiza el desarrollo integral y holístico del profesional en aspectos que se traduzcan en atención de alto valor, eficiencia y seguridad para el paciente. Algunos ejemplos de estos marcos de competencia profesional son el Accreditation Council for Graduate Medical Education (ACGME) / American Board of Medical Specialties de los Estados Unidos, el General Medical Council (GMC) del Reino Unido (Tomorrows' doctors) y el del Royal College of Physicians and Surgeons (RCPSC) (CanMEDS) de Canadá ${ }^{16-18}$. Igualmente, los hallazgos del consenso son consistentes con los esfuerzos nacionales para la transformación de la educación médica, realizados por la Comisión para la Transformación de la Educación Médica en Colombia ${ }^{9}$.

Respecto al segundo hallazgo, este consenso enfatiza la necesidad de competencia práctica y destreza operatoria del cirujano egresado de los programas colombianos en diferentes procedimientos que abarcan múltiples áreas de la cirugía. Existen varias razones que sustentan esta necesidad. Para el año 2018, Colombia tiene una población de 48,2 millones de habitantes, el número total de cirujanos en el país es de 2475 y la relación poblacional es de 5 cirujanos/ 100.000 habitantes ${ }^{19}$, que es una relación aún distante de la densidad esperada para la fuerza laboral en cirugía, según la Comisión Lancet para la Cirugía Global que recomienda 20-40 cirujanos / 100.000 habitantes ${ }^{20}$. Los cirujanos en Colombia, por lo tanto, parecen estar trabajando mas allá de su capacidad, si se considera que realizan entre 2690 y 3090 procedimientos / 100.000 habitantes por año ${ }^{19}$. Adicionalmente, es claro que la distribución del recurso humano en cirugía en el país no es óptima y que la oferta de sub-especialistas es limitada ${ }^{19,20}$.
En tercer lugar, el perfil demográfico y epidemiológico del país exige que los cirujanos estén bien entrenados para atender condiciones que continúan en ascenso, como el trauma, el cáncer y las infecciones ${ }^{19,21,22}$. No obstante, aunque todas estas consideraciones son válidas, de acuerdo con la percepción de los profesores de los programas de cirugía del país, no se reportó consenso en casi un tercio de los procedimientos quirúrgicos declarados por los programas.

Posiblemente, las explicaciones para este fenómeno se encuentran en las limitaciones para el entrenamiento propias de los programas (por infraestructura, recurso humano o volumen operatorio), que impiden garantizar que los residentes participen en un número suficiente de procedimientos oncológicos y mínimamente invasivos, en especial gastrointestinales y colorrectales, en el marco de la seguridad del paciente. También, en el crecimiento acelerado de grupos especializados en estas áreas de la cirugía, especialmente en Bogotá y Medellín, en donde se concentran la mayoría de los programas. Este hallazgo, aparentemente contradictorio, representa un llamado de atención para fortalecer el entrenamiento de los cirujanos de acuerdo con el perfil epidemiológico de las regiones del país, y para incrementar la cooperación universitaria y el trabajo entre los programas.

Igualmente, la ausencia de consenso en el 26,7 $\%$ de los procedimientos invita a revisar periódicamente estos hallazgos para generar nuevos acuerdos, en función del advenimiento tecnológico, crecimiento del recurso humano, infraestructura y capacidad de los programas. Al respecto, vale la pena recordar que el alcance de este este consenso es el "acuerdo sobre los mínimos" procedimientos que debe realizar un egresado de cualquier programa de cirugía colombiano, de forma autónoma, independiente y sin supervisión; por lo tanto, es una evaluación transversal que amerita revisión permanente.

Finalmente, este consenso enfatiza las APC, como uno de los ejes centrales de la educación quirúrgica basada en competencia para el país. Este aspecto es importante pues permite trasladar el marco convencional de competencias a la 
práctica. En otras palabras, permite ir un paso mas allá de las competencias, para describir y evaluar actividades especificas de desempeño profesional en un entorno real de trabajo ${ }^{23}$.

Este consenso identifica cuatro actividades principales que describen un amplio ámbito de ejercicio profesional del cirujano general colombiano, dirigidas a varios grupos de pacientes, en diferentes contextos y situaciones de práctica. Estos hallazgos se suman al reciente debate sobre el rol de las APC en cirugía, originado en los Países Bajos, que constituye el centro de la educación quirúrgica contemporánea, y en especial en Norte América ${ }^{5-8}$. Esta conversación académica, sin embargo, es limitada aún en América Latina.

En este contexto, la Asociación Colombiana de Cirugía es pionera en el desarrollo de un marco curricular nacional para una especialidad médicoquirúrgica. El presente estudio, por lo tanto, representa un aporte original a la educación médica del país, tiene implicaciones para el entrenamiento de los futuros cirujanos y se suma al escaso cuerpo de evidencia sobre las APC en la cirugía colombiana ${ }^{24-26 .}$

Este estudio cuenta con varias fortalezas, entre ellas, que el consenso fue desarrollado con la participación de la mitad de los profesores de los programas de los veinte programas de Especialización en Cirugía General en Colombia. La validez de contenido del cuestionario fue establecida a partir de la revisión de un extenso número de fuentes primarias (documento maestro registrado ante el Ministerio de Educación), en los que cada programa declara al país su perspectiva para la formación de cirujanos generales. Además, el consenso se desarrolló a través de un método formal y riguroso, que involucró recomendaciones estándar (método Delphi).

No obstante, el estudio posee algunas limitaciones. En primer lugar, se evaluó únicamente la perspectiva de la comunidad académica. Si bien, las universidades son las encargadas de la formación del recurso humano especializado en salud en Colombia, en este consenso la perspectiva de otros actores es limitada. En segundo lugar, el consenso contó con la participación preponderante de cirujanos provenientes de programas de especialización ubicados en la ciudad de Bogotá. En tercer lugar, la percepción de los cirujanos especialistas fue escasa.

Las limitaciones mencionadas, sin embargo, ofrecen la oportunidad de realizar nuevos estudios. Rescatando el valor de este primer paso para el dialogo académico sobre un currículo nacional de la especialidad, en las revisiones futuras del consenso se debe contar con la participación de otros actores, por ejemplo, cirujanos no académicos, aseguradores y administradores hospitalarios, para ampliar la mirada del ejercicio de la profesión. Es necesario conducir nuevos estudios que exploren a profundidad la perspectiva de cirujanos provenientes de otras regiones, diferentes a la capital del país, revisar el consenso y discutirlo con cirujanos especialistas, y traducir estos contenidos en un plan de estudio (cuya duración y organización es difícil de establecer en el presente consenso) y métodos de evaluación del desempeño profesional del residente.

Este consenso tiene importantes implicaciones para la práctica educativa, pues sirve como marco de referencia para la creación de programas y renovación de registros calificados y procesos de acreditación de los programas existentes. También es una referencia para el registro nacional de procedimientos quirúrgicos en los que participan los residentes. Igualmente, es una referencia para optimizar la capacidad de los programas, especialmente en situaciones de contingencia, por lo que se alinea con las recomendaciones de la Asociación Colombiana de Cirugía para la educación en pandemia por SARS/CoV-2 ${ }^{27}$. Finalmente, es un marco de referencia para otras asociaciones científicas, nacionales e internaciones, interesadas en desarrollar marcos curriculares generales a nivel nacional, así como para fortalecer el dialogo académico universitario y la cooperación entre programas.

En conclusión, el presente consenso contribuye a la creación de un currículo nacional para la Especialización en Cirugía General en Colombia, tiene implicaciones prácticas y ofrece oportunidades de investigación futura. 


\section{Cumplimiento de normas éticas:}

Consentimiento informado: Esta publicación no involucra pacientes. Los participantes contestaron la encuesta de manera libre y voluntaria, y como tal no hay necesidad de un consentimiento informado ni de aprobación de un Comité de Ética Institucional.

Conflicto de intereses: Ninguno declarado por los autores.

Fuentes de financiación: Elaborado con el apoyo de la División de Educación de la Asociación Colombiana de Cirugía y recursos propios de los autores.

\section{Contribución de los autores}

- Concepción y diseño del estudio: Luis Carlos Domínguez Álvaro Enrique Sanabria, Lilian TorregrosaAlmonacid.

- Adquisición de datos: Luis Carlos Domínguez, Álvaro Enrique Sanabria.

- Análisis e interpretación de datos: Luis Carlos Domínguez Álvaro Enrique Sanabria, Lilian TorregrosaAlmonacid, William Sánchez, Neil Valentín Vega, Felipe Vargas, Álvaro Niño, Liliana Cuevas, Sergio Iván Hoyos, Luis Gerardo Cadavid, German Jiménez, Julián Sarmiento, Mauricio Osorio, Maikel Pacheco, Rubén Caicedo, Rolando Medina, Jimmy León, Mauricio Zuluaga, Hernando Coba, Erwin Rodríguez, Carlos Luna-Jaspe, Jaime Merlano, Juan Jacobo Molina, Carlos Bustillo.

- Redacción del manuscrito: Luis Carlos Domínguez, Álvaro Enrique Sanabria, Lilian Torregrosa-Almonacid, William Sánchez, Neil Valentín Vega, Felipe Vargas, Álvaro Niño, Liliana Cuevas, Sergio Iván Hoyos, Luis Gerardo Cadavid, German Jiménez, Julián Sarmiento, Mauricio Osorio, Maikel Pacheco, Rubén Caicedo, Rolando Medina, Jimmy León, Mauricio Zuluaga, Hernando Coba, Erwin Rodríguez, Carlos Luna-Jaspe, Jaime Merlano, Juan Jacobo Molina, Carlos Bustillo.

- Revisión crítica: Luis Carlos Domínguez,Álvaro Enrique Sanabria, Lilian Torregrosa-Almonacid, William Sánchez, Neil Valentín Vega, Felipe Vargas, Álvaro Niño, Liliana Cuevas, Sergio Iván Hoyos, Luis Gerardo Cadavid, German Jiménez, Julián Sarmiento, Mauricio Osorio, Maikel Pacheco, Rubén Caicedo, Rolando Medina, Jimmy León, Mauricio Zuluaga, Hernando Coba, Erwin Rodríguez, Carlos Luna-Jaspe, Jaime Merlano, Juan Jacobo Molina, Carlos Bustillo.

\section{Referencias}

1. Frank JR, Mungroo R, Ahmad Y, Wang M, De Rossi S, Horsley T. Toward a definition of competency-based education in medicine: A systematic review of published definitions. Med Teach. 2010;32:631-7. https://doi.org/10.3109/0142159X.2010.500898

2. Epstein RM, Hundert EM. Defining and assessing professional competence. JAMA. 2002;287:226-35. https://doi.org/10.1001/jama.287.2.226

3. Albanese MA, Mejicano G, Mullan P, Kokotailo P, Gruppen L. Defining characteristics of educational competencies. Med Educ; 2008;42:248-55. https://doi.org/10.1111/j.1365-2923.2007.02996.x

4. ten Cate 0 . Nuts and bolts of entrustable professional activities. J Grad Med Educ. 2013;5:157-8. https://doi.org/10.4300/JGME-D-12-00380.1

5. Stucke RS, Sorensen M, Rosser A, Sullivan S. The surgical consult entrustable professional activity (EPA): Defining competence as a basis for evaluation. Am J Surg. 2020;219:253-7.

https://doi.org/10.1016/j.amjsurg.2018.12.056

6. Stahl CC, Jung SA, Rosser AA, Kraut AS, Schnapp BH, Westergaard $\mathrm{M}$, et al. Entrustable professional activities in general surgery: Trends in resident self-assessment. J Surg Educ. 2020;77:1562-7. https://doi.org/10.1016/j.jsurg.2020.05.005

7. Stahl CC, Collins E, Jung SA, Rosser AA, Kraut AS, Schnapp $\mathrm{BH}$, et al. Implementation of entrustable professional activities into a general surgery residency. J Surg Educ. 2020;77:739-48. https://doi.org/10.1016/j.jsurg.2020.01.012

8. Brasel KJ, Klingensmith ME, Englander R, Grambau M, Buyske J, Sarosi G, Minter R. Entrustable professional activities in general surgery: Development and implementation. J Surg Educ. 2019;76:1174-86.

https://doi.org/10.1016/j.jsurg.2019.04.003

9. Republica de Colombia. Ministerio de Salud y Protección Social - Ministrerio de Educación Nacional. Documento de recomendaciones para la transformación de la educacion medica en Colombia. Fecha de consulta: Diciembre 3 de 2020. Disponible en: https://www.minsalud.gov.co/sites/rid/Lists/BibliotecaDigital/RIDE/ VS/transformacion-educacion-medica-colombia.pdf

10. Republica de Colombia. Ministerio de Salud y Protección Social. Dirección de Desarrollo del Talento Humano en Salud. Política nacional de talento humano en salud. 2018. Fecha de consulta: Diciembre 6 de 2020. Disponible en: https://www.minsalud.gov.co/sites/ rid/Lists/BibliotecaDigital/RIDE/VS/TH/politica-nacional-talento-humano-salud.pdf

11. Humphrey-Murto S, Varpio L, Wood TJ, Gonsalves C, Ufholz LA, Mascioli K, et al. The Use of the Delphi and other consensus group methods in medical education research: A review. Acad Med. 2017;92:1491-8. https://doi.org/10.1097/ACM.0000000000001812 
12. de Villiers MR, de Villiers PJT, Kent AP. The Delphi technique in health sciences education research. Med Teach. 2005;27:639-43. https://doi.org/10.1080/13611260500069947

13. Diamond IR, Grant RC, Feldman BM, Pencharz PB, Ling SC, Moore AM, Wales PW. Defining consensus: A systematic review recommends methodologic criteria for reporting of Delphi studies. J Clin Epidemiol. 2014;67:401-9. https://doi.org/10.1016/j.jclinepi.2013.12.002

14. Departamento Administrativo Nacional de Estadística (DANE). Geoportal. Fecha de consulta: Diciembre 6 de 2020. Disponible en: https://geoportal.dane.gov.co/

15. Batt AM, Tavares W, Williams B. The development of competency frameworks in healthcare professions: a scoping review. Advances in Health Sciences Education. 2020;25:913-87. https://doi.org/10.1007/s10459-019-09946-w

16. Chou S, Cole G, McLaughlin K, Lockyer J. CanMEDS evaluation in Canadian postgraduate training programmes: Tools used and programme director satisfaction. Med Educ. 2008;42:879-86. https://doi.org/10.1111/j.1365-2923.2008.03111.x

17. Rubin P, Franchi-Christopher D. New edition of Tomorrow's Doctors. Med Teach. 2002;24:368-9. https://doi.org/10.1080/0142159021000000816

18. Englander R, Cameron T, Ballard AJ, Dodge J, Bull J, Aschenbrener CA. Toward a common taxonomy of competency domains for the health professions and competencies for physicians. Acad Med. 2013;88:1088-94. https://doi.org/10.1097/ACM.0b013e31829a3b2b

19. Hanna JS, Herrera-Almario GE, Pinilla-Roncancio M, Tulloch D, Valencia SA, Sabatino ME, et al. Use of the six core surgical indicators from the Lancet Commission on Global Surgery in Colombia: a situational analysis. Lancet Glob Health. 2020;8:e699-710. https://doi.org/10.1016/S2214-109X(20)30090-5

20. Meara JG, Leather AJM, Hagander L, Alkire BC, Alonso N, Ameh EA, et al. Global Surgery 2030: Evidence and solutions for achieving health, welfare, and economic development. Lancet. 2015;386:569-624.

http://dx.doi.org/10.1016/S0140-6736(15)60160-X

21. Raykar NP, Yorlets RR, Liu C, Greenberg SLM, Kotagal M, Goldman R, et al. A qualitative study exploring contextual challenges to surgical care provision in 21 LMICs. Lancet. 2015;385(Suppl 2):S15.

https://doi.org/10.1016/S0140-6736(15)60810-8

22. Bustos Y, Castro J, Wen LS, Sullivan AF, Chen DK, Camargo CA. Emergency department characteristics and capabilities in Bogotá, Colombia. Int J Emerg Med. 2015;8:79.

https://doi.org/10.1186/s12245-015-0079-y

23. Cate OT, Hart D, Ankel F, Busari J, Englander R, Glasgow $\mathrm{N}$, et al. Entrustment decision making in clinical training. Acad Med. 2016;91:191-8. https://doi.org/10.1097/ACM.0000000000001044

24. Domínguez LC, Urueña N, Sanabria Á, Pepín JJ, Mosquera M, Vega V, Osorio C. Autonomía y supervisión del residente de cirugía: ¿se cumplen las expectativas en el quirófano? Educ Médica. 2018;19:208-16.

25. Senociain JE, Domínguez LC. Transferencia de autonomía operatoria en apendicectomía abierta y laparoscópica: Un estudio de métodos mixtos desde la perspectiva de residentes y supervisores. Rev Colomb Cir. 2020;35:558-69. https://doi.org/10.30944/20117582.551

26. Moros G, Domínguez LC, Sierra D, Villarraga A, Pepin JJ. Actividades profesionales confiables en cirugia minimamente invasiva: iniciando el camino para lograr las competencias en el futuro graduado de medicina. Rev Chil Cir. 2018;70:503-9.

27. Dominguez Torres LC, Torregrosa Almonacid L, Sánchez Maldonado W, Lasprilla N, Vargas Barato F, Niño Rodríguez ÁE, et al. Educación quirúrgica durante la Pandemia COVID-19: Primer consenso nacional de la División de Educación de la Asociación Colombiana de Cirugía. Rev Colomb Cir. 2020;35:363-72. https://doi.org/10.30944/20117582.685 